\title{
CAMP/PKA signaling balances respiratory activity with mitochondria dependent apoptosis via transcriptional regulation
}

\author{
Jane E Leadsham, Campbell W Gourlay
}

\begin{abstract}
Background: Appropriate control of mitochondrial function, morphology and biogenesis are crucial determinants of the general health of eukaryotic cells. It is therefore imperative that we understand the mechanisms that coordinate mitochondrial function with environmental signaling systems. The regulation of yeast mitochondrial function in response to nutritional change can be modulated by PKA activity. Unregulated PKA activity can lead to the production of mitochondria that are prone to the production of ROS, and an apoptotic form of cell death.

Results: We present evidence that mitochondria are sensitive to the level of CAMP/PKA signaling and can respond by modulating levels of respiratory activity or committing to self execution. The inappropriate activation of one of the yeast PKA catalytic subunits, Tpk3p, is sufficient to commit cells to an apoptotic death through transcriptional changes that promote the production of dysfunctional, ROS producing mitochondria. Our data implies that CAMP/ PKA regulation of mitochondrial function that promotes apoptosis engages the function of multiple transcription factors, including HAP4, SOK2 and SCO1.

Conclusions: We propose that in yeast, as is the case in mammalian cells, mitochondrial function and biogenesis are controlled in response to environmental change by the concerted regulation of multiple transcription factors. The visualization of CAMP/TPK3 induced cell death within yeast colonies supports a model that PKA regulation plays a physiological role in coordinating respiratory function and cell death with nutritional status in budding yeast.
\end{abstract}

\section{Background}

Mitochondria participate in a number of essential cellular functions, for example they are key players in ATP production, via the process of oxidative phosphorylation, which can produce up to 15 times more ATP from glucose than glycolysis alone. They are also central to metabolic regulation and facilitate diverse cell signaling events [1,2]. Mitochondria are therefore essential for the maintenance, adaptability and survival of eukaryotic cells. In addition, these remarkable organelles have been conclusively shown to play a role in the regulation of programmed cell death processes (reviewed in [3]), and act as an important determinants of cellular senescence and ageing $[4,5]$. The importance of understanding how mitochondria influence the metabolic status of cells

\footnotetext{
* Correspondence: C.W.Gourlay@kent.ac.uk
Department of Biosciences, University of Kent, Canterbury Kent, CT2 7NJ,

* Correspondence: C.W.Gourlay@kent.ac.uk
Department of Biosciences, University of Kent, Canterbury Kent, CT2 7NJ, England, UK
}

(c) 2010 Leadsham and Gourlay; licensee BioMed Central Ltd. This is an Open Access article distributed under the terms of the Creative Commons Attribution License (http://creativecommons.org/licenses/by/2.0), which permits unrestricted use, distribution, and reproduction in any medium, provided the original work is properly cited. lar and neurodegenerative diseases have been linked with their dysfunction. The current list of disease pathologies in which mitochondrial function is thought to be a major contributing factor is extensive and includes diabetes [6], cancer [7], multiple sclerosis [8], Alzheimer's [9] and Parkinson's [10]. Good evidence also exists that indicates a loss of mitochondrial function occurs during the progression of normal ageing [11]. Mitochondrial dysfunction can lead to the production of reactive oxygen species (ROS), which are implicated in both ageing and apoptosis [12,13], presumably as a result of their ability to damage macromolecules.

The control of mitochondrial biogenesis is complex, requiring coordinated transcription of a large number of nuclear and mitochondria encoded genes. In addition, there must be concerted control of the synthesis, import, and incorporation of proteins and lipids to 
existing mitochondria alongside appropriate replication of the mitochondrial DNA (mtDNA). In mammalian cells a number of have been indentified that act to coordinate tissue specific mitochondrial biogenesis in the face of altered metabolic demand and environmental change, including; nuclear respiratory factor-1 (NRF-1) and GA-binding protein (GABP or NRF-2), peroxisome proliferator-activated receptors (PPAR $\alpha, \mathrm{PPAR} \delta$, and PPAR $\gamma$ ), Mitochondrial transcription factors TFAM, TFB1M and TFB2M, Estrogen-related receptors (ERR $\alpha$, ERR $\beta$, and ERR $\gamma$ ) (for a recent review see [14]). The mitochondria of yeast are also adapted to respond to rapidly changing metabolic demands. For example switching between fermentative and non-fermentative carbon sources requires adjustment of mitochondrial activity and density [15]. However despite the importance of the mitochondria to cellular function, the signaling mechanisms by which their activity is coordinated with environmental conditions in yeast are not well documented.

Previous studies in yeast have established links between Ras signaling and mitochondrial function, via cAMP/PKA dependent and independent routes $[16,17]$. Within yeast Ras/cAMP/PKA signaling also controls cellular processes that include cell growth and proliferation and the induction of stress responses [18], making this pathway a good candidate to integrate environmental signaling with mitochondrial regulation. In yeast, protein kinase A (PKA) consists of a single regulatory subunit encoded by the $B C Y 1$ gene [19] and three catalytic subunits Tpk1p, Tpk2p and Tpk3p [20]. PKA is activated by an increase in cAMP concentration, which is generated in the cell from adenylyl cyclase, Cyr1p [21]. The three catalytic subunits of yeast exhibit high similarity and have been shown to have both specific and overlapping functions. For instance Tpk $2 p$ has been shown to influence iron uptake, trehalase synthesis, water homeostasis [22] and pseudohyphal growth [23]. Tpk1p has been implicated in the branched chain amino acid biosynthesis pathway, mitochondrial iron homeostasis and mtDNA stability [22]. Excessive PKA activity can prove deleterious, for instance the overexpression of TPK3 has been shown to inhibit growth [24]. An important regulator of Cyr1p activation is the small regulatory GTPase Ras [21]. The regulation of cAMP production from Cyr1p in response to Ras activation also requires the protein Srv2p/CAP $[25,26]$, which is able to bind both adenylyl cyclase and actin structures. We have shown previously that the accumulation of stable actin aggregates leads to the hyperactivation of the Ras/ cAMP/PKA pathway [27]. The result of actin aggregation induced Ras/cAMP/PKA signaling is that ROS are produced from dysfunctional mitochondria, facilitating cell death that displays hallmarks of yeast apoptosis [27-29]. The deletion of TPK3 is sufficient to prevent the production of ROS in actin aggregating strains, implicating this PKA subunit as a regulator of mitochondrial function $[27,30]$. Further evidence for a role of Tpk3p in the regulation of mitochondrial function comes from a study in which cells lacking Tpk3p showed reduced respiratory activity [31].

Here we demonstrate that increased Tpk3p activity is sufficient to induce formation of dysfunctional mitochondria with striking morphological abnormalities that produce high levels of ROS. We provide evidence that the loss of mitochondria function and ROS production associated with elevated Tpk3p activity arises as a result of transcriptional changes that inhibit mitochondrial biogenesis, corrupt the electron transport chain and inhibit stress response mechanisms. We also show that the loss of mitochondrial function and production of ROS requires the activity of the transcriptional regulators HAP4, SOK2 and SKO1. This paper therefore establishes important links between cAMP/PKA signaling, nutritional sensing, mitochondrial biogenesis and ROS production.

\section{Methods}

Yeast strains, plasmids, Media and Growth Conditions

All yeast strains used in this work are derivatives of BY4741 and are listed in Table 1. Unless stated otherwise, cells were grown in a rotary shaker at $30^{\circ} \mathrm{C}$ in synthetic complete medium or synthetic medium lacking an appropriate nutrient for auxotrophic selection. PDE2 was disrupted using the loxP marker cassette system [32]. Oligonucleotides designed for the targeted disruption of genes are described in Additional File 1. Plasmids used to overexpress TPK3 [33] and HAP4 [34] have also been described previously.

\section{RNA Isolation and Affymatrix Microarray Procedure}

Strains CGY502 ( $\Delta p d e 2)$ and CGY217 ( $\Delta p d e 2 \Delta t p k 3)$ were grown for 24 hours in YPD (1\% yeast extract, $2 \%$ Bacto-peptone, $2 \%$ glucose) supplemented with $4 \mathrm{mM}$ cAMP at $30^{\circ} \mathrm{C}$ in a rotary air incubator. Cells from triplicate $20 \mathrm{ml}$ cultures were harvested by centrifugation and resuspended in a small volume of media. The suspension was dispensed dropwise into liquid nitrogen. Triplicate cell pellets were maintained on dry ice and sent to the COGEME Transcriptome facility at the University of Manchester http://cogeme.ex.ac.uk/index.html for RNA isolation and microarray analysis. A yeast 2 Affymetrix array was used in these experiments and statistical analysis on a gene by gene basis carried out using Limma software. Quality Control analysis was carried out using dchip software. Raw and normailsed data from the microarray has been deposited in the Gene 
Table 1 Yeast strains used in this study

\begin{tabular}{|c|c|c|}
\hline Strain & Genotype & Origin \\
\hline CGY424 & mata his3 $\Delta 1$ leu2 $\Delta$ met15 $\Delta$ ura3 $\Delta$ & Research Genetics \\
\hline CGY502 & mata his3 $\Delta 1$ leu2 $\Delta$ met15 $\Delta$ ura3 $\Delta$ spde2::KanMx & Research Genetics \\
\hline CGY217 & 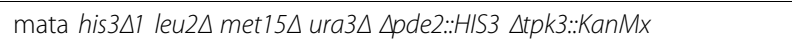 & {$[27]$} \\
\hline CGY215 & mata his3 $\Delta 1$ leu2 $\Delta$ met15 $\Delta$ ura3 $\Delta$ pde2::HIS3 $\Delta$ tpk1::KanMx & [27] \\
\hline CGY216 & mata his3 $\Delta 1$ leu2 $\Delta$ met15 $\Delta$ ura3 $\Delta$ spde2::HIS3 $\Delta t p k 2:: K a n M x$ & This study \\
\hline CGY807 & 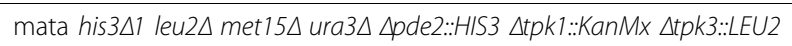 & This study \\
\hline CGY808 & 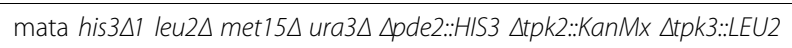 & This study \\
\hline CGY819 & 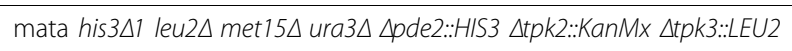 & This study \\
\hline CGY638 & mata his3 $\Delta 1$ leu2 $\Delta$ met15 $\Delta$ ura3 $\Delta \triangle$ cox4:.KanMx & This study \\
\hline
\end{tabular}

Expression Omnibus (GEO) database, accession number GSE25541.

\section{High Resolution Respirometry}

Intact cell respiration was determined at $30^{\circ} \mathrm{C}$ using an Oxygraph-2 k system (Oroboros, Innsbruck, Austria) equipped with two chambers. Data was analysed using DatLab software. Yeast cells $(2 \mathrm{ml})$ at a concentration of $3.5 \times 10^{6} / \mathrm{ml}$, in minimal media without glucose, were added to each chamber. All assays were conducted in biological triplicate. The chambers were closed and Routine respiration was recorded. LEAK respiration was determined by the addition of $150 \mu \mathrm{M}$ TET (Sigma), an ATP synthase inhibitor. Uncoupled respiration was then determined by the addition of the ionophore FCCP (12 $\mu \mathrm{M})$ (Sigma). The addition of $2 \mu \mathrm{M}$ Antimycin A (Sigma) accounted for non-mitochondrial oxygen consumption.

\section{Immunoblotting}

Cell number was accurately determined using a haemocytometer and total protein extracted using as optimized protein extraction for method suitable for quantitative proteomics [35]. Samples were separated by SDS PAGE and transferred to PVDF membranes before probing with primary antibodies at the final concentrations, antiCox4 (mitoscience 1: 1000), anti-Cox2 (mitoscience 1:1000), anti-Por1 (Molecular Probes, Invitrogen 1:500) and anti-actin (1:2000).

\section{Fluorescence microscopy}

Rhodamine-phalloidin and DAPI staining were performed as previously described for F-actin [36]. Cells were viewed with an Olympus IX-81 fluorescence microscope with a $150 \mathrm{~W}$ Xenon/mercury lamp and an Olympus 150x Plan NeoFluor oil-immersion objective. GFP/RFP Co-localisation studies were performed using an Optosplit II Image Splitter (Cairn Scientific). Images were captured using a Hammamatsu ORCA AG digital camera using Olympus Cell R software.

\section{Analysis of mitochondria}

Assessment of reactive oxygen species content and mitochondrial membrane potential were carried out as previously described [27].

GFP labeled mitochondria were visualized using the plasmids pVTU100U-mtGFP and pYX122-mtGFP [37].

\section{Analysis of cell death in yeast colonies}

Between 10 and 20 cells were plated onto $9 \mathrm{~cm}$ YPAD agar plates containing $10 \mu \mathrm{M}$ Phloxine B (Sigma). Colonies were grown for five days before being visualized using a Leica M2FLIII microscope and documented with a Leica DC300F colour camera. To visualise a cross section through the colony, a glass coverslip was inserted directly, bisecting the colony, and removed from the agar. Dissected colonies were visualized and documented immediately. All images were taken using the same illumination conditions and exposure times.

\section{Glycogen staining}

Iodine staining of yeast cells as an assay for glycogen content was carried out as previously described (Care et al., 2004).

\section{Results}

CAMP/PKA regulation of respiratory function

Our previous studies have shown that aggregation of the actin cytoskeleton results in hyperactivation of the Ras/ cAMP/PKA signaling pathway, leading to the loss of respiratory function, ROS production and an apoptotic cell death [27]. The $S$. cerevisiae genome encodes for three PKA subunits, encoded by TPK1, TPK2 and TPK3. In order to establish which PKA subunits were required to induce the loss of respiration and ROS production, we generated a series of mutant strains expressing either a single PKA isoform, or combinations of multiple subunit encoding genes. Using this genetic approach we were able to assess the influence of individual PKA isoforms on respiratory function during normal growth, or under conditions of elevated cAMP. Elevation of cAMP levels 
was achieved by the deletion of the high affinity cAMP phosphodiesterase, $P D E 2$, which catalyses the breakdown of cAMP. The loss of Pde2p function allows manipulation of levels of this secondary messenger by exogenous cAMP addition [38]. We performed high resolution respirometry on PKA mutant strains grown for $24 \mathrm{~h}$ to diauxic shift in the presence or absence of $4 \mathrm{mM}$ cAMP, using an Oroboros Oxygraph, which accurately measures changes in oxygen concentration in a sealed system. By measuring the fall in oxygen concentration within the chamber the oxygen flux can be determined. Oxygen consumption, which occurs at the Cytochrome c Oxidase complex (complex IV), is a direct measure of electron transport system (ETS) activity. Using this sensitive technique it was possible to detect a significant reduction in respiratory activity in wild type cells grown in the presence of exogenous cAMP (Figure 1A). Interestingly, the loss of PDE2 led to a significant increase in respiration, while addition of further cAMP

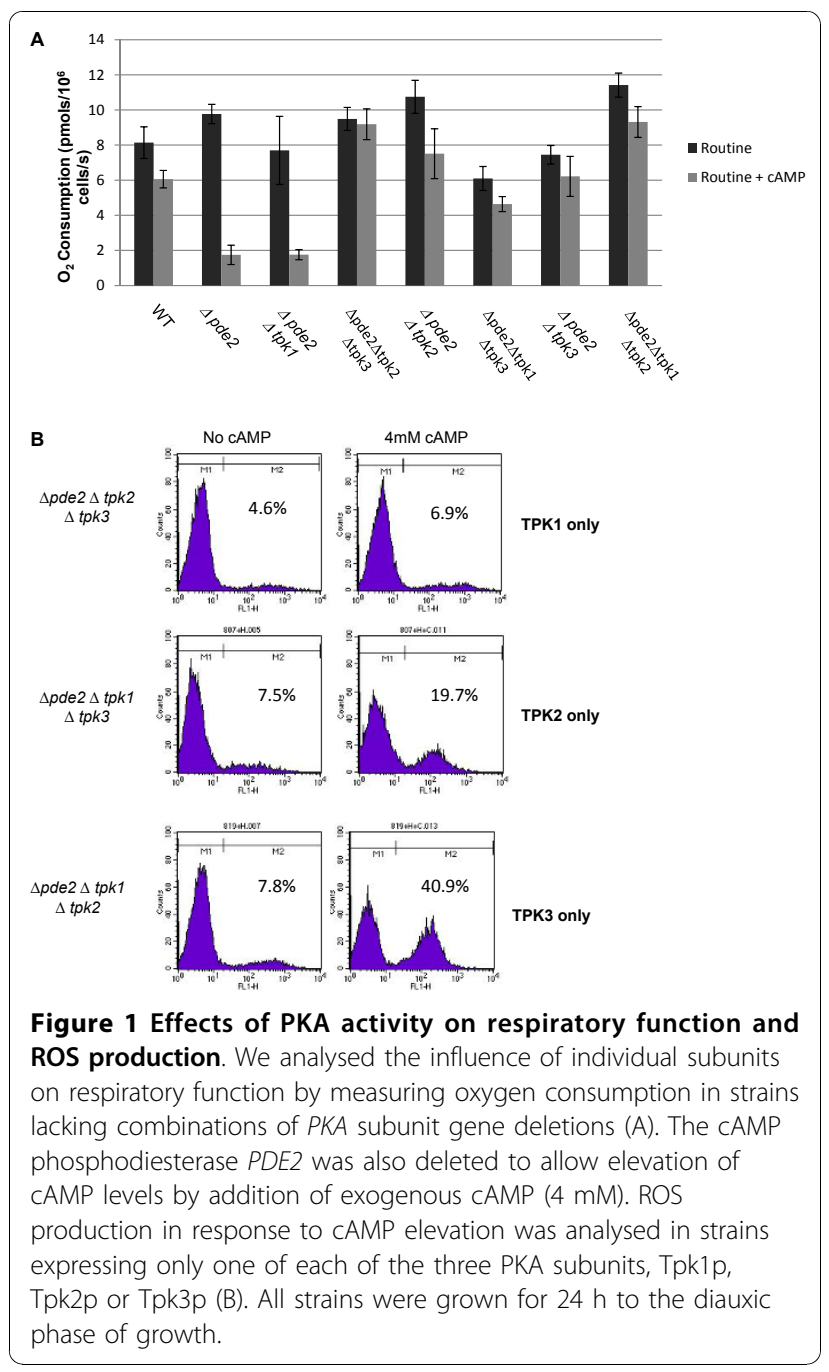

in this background led to a dramatic reduction in oxygen consumption. These results suggest that the level of respiratory activity is modulated in response to variation of intracellular cAMP levels.

The addition of cAMP to cells lacking both PDE2 and the TPK1 subunit genes led to a reduction in respiration similar to that observed in the $\Delta p d e 2$ single mutant. However while the level of routine respiration differed between strains (Figure 1A), triple mutant $\Delta p d e 2 \Delta t p k 2 \Delta t p k 3, \Delta p d e 2 \Delta t p k 1 \Delta t p k 3$ or $\Delta p d e 2 \Delta$ tpk1 1 tpk2 strains did not exhibit the collapse of respiratory activity observed $\Delta p d e 2$ or $\Delta p d e 2 \Delta \mathrm{tpk} 1$ cells when cAMP levels were elevated (Figure 1A). Similarly $\Delta p d e 2 \Delta$ tpk 2 or $\Delta p d e 2 \Delta t p k 3$ double mutant cells were resistant to the effects of elevated cAMP. Collectively these data suggest that the collapse of respiration observed in $\triangle p d e 2$ cells when grown in the presence of exogenous cAMP requires the presence of both TPK2 and TPK3, but occurs independently of TPK1.

We then examined the accumulation of ROS in response to cAMP elevation within cell populations expressing Tpk1p, Tpk2p or Tpk3p as their sole source of PKA activity (Figure 1B). We found that the elevation of cAMP in strains expressing TPK1 only resulted in no difference in the number of cells producing high ROS levels within a population (Figure 1B). In contrast, cells expressing TPK2 or TPK 3 only did show an increase in the number of ROS producing cells when cAMP levels were increased (Figure 1B). These data are in accordance with the findings in Figure 1A, that both TPK2 and TPK3 play a role in the regulation of respiratory activity in response to cAMP elevation. However it should be noted that cells expressing TPK3 only displayed the most significant increase in the number of ROS producing cells (Figure 1B), indicating that this PKA subunit may play a more fundamental role in the regulation of radical production.

\section{Tpk3p activity is sufficient to induce respiratory collapse and ROS production}

Although TPK2 and TPK3 would appear to be essential to induce the collapse of respiration under conditions of elevated cAMP (Figure 1A), our previous data points to the loss of Tpk3p as sufficient to prevent ROS production from the mitochondria when cAMP levels are raised [27]. We therefore sought to characterise in detail the mitochondrial defects associated with elevated Tpk3p activity. Wild type, $\Delta p d e 2, \Delta p d e 2 \Delta t p k 3$ and $\Delta p d e 2 \Delta t p k 3+T P K 3$ strains were cultured for 24 hours in media supplemented with $2 \%$ glucose to the diauxic shift phase of growth. During the diauxic shift cells sense a reduction in glucose and prepare to shift to a non-fermentative metabolism, which requires functional mitochondria. At this stage the basal, or routine, level of 


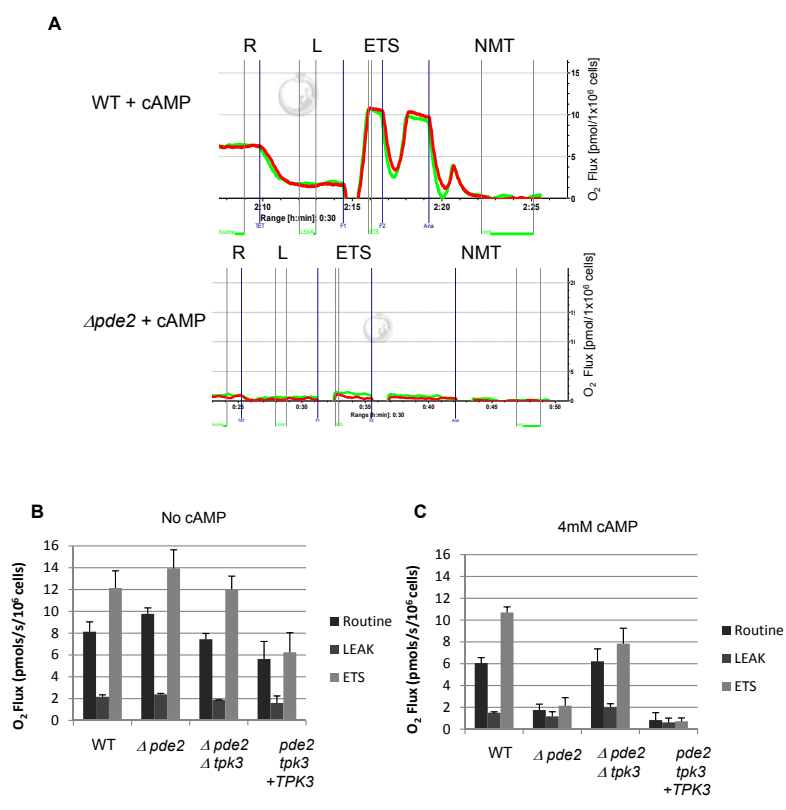

Figure 2 Excessive Tpk3p activity leads to loss of mitochondrial respiration. Cells were grown for 24 hours to diauxic shift in minimal medium in the presence or absence of 4 mM cAMP. Mitochondrial respiration was measured using an Oroboros high resolution respirometer. Typical traces from two biological replicates (red and green lines) wild type and $4 p d e 2$ cells grown in the presence of $4 \mathrm{mM}$ CAMP are shown (A). Routine respiration (designated by $\mathrm{R}$ ) represents oxygen consumption from cells placed in the respirometer immediately from culture and without drugs. The addition of TET, an ATPsynthase inhibitor, causes proton build up on the outside of the inner mitochondrial membrane, inhibiting respiration. Respiration that does occur happens as a result of the small LEAK of protons across the inner membrane (designated by $L$ ). Dissipation of this proton gradient by the proton ionophore FCCP restores mitochondrial respiration (designated by ETS) but removes the major control point, being the proton gradient which is controlled by the rate of oxidative phosphorylation. The final addition of Antimycin A blocks activity of complex III, preventing electrons moving to complex IV and therefore oxygen consumption is halted and non-mitochondrial oxygen consumption (designated by NMT) is accounted for. The oxygen consumption under these conditions was determined for strains wild type, $\triangle$ pde2, $\triangle$ pde $2 \Delta$ tpk3 and $\triangle$ pde $2 \triangle$ tpk3 + TPK3 grown in the absence (B) and presence (C) of $4 \mathrm{mM}$ cAMP. Error bars are the standard error of 3 independent experiments.

respiration was assessed (Figure 2A, marked R). Following stabilisation of routine respiration, the ATPsynthase/ Complex V inhibitor, triethyltin bromide (TET) was used to determine LEAK flux (Figure 2A. marked L) which represents non-phosphorylating respiration due to proton leakage back across the Inner Mitochondrial Membrane. Oligomycin is commonly used for this purpose [39] but we found that the BY4741 background was resistant to its effects. TET can also be utilised to inhibit complex V [40] and following titration studies a concentration of $150 \mu \mathrm{M}$ was found to be effective for this purpose in our strain background. Following treatment with TET, cells were exposed to $12 \mu \mathrm{M}$ FCCP, a protonophore, which is able to dissipate the mitochondrial membrane potential. The proton gradient is a major control point of the ETS and its removal allows quantification of the maximum capacity of the ETS (Figure 2A. marked ETS). A second dose of $3 \mu \mathrm{M}$ FCCP (seen as a second peak Figure 2A.) was administered to demonstrate that $12 \mu \mathrm{M}$ FCCP was sufficient for complete uncoupling and the induction of maximal respiration. Finally, cells were exposed to the complex III inhibitor Antimycin A which prevents all mitochondrial related oxygen consumption (Figure 2A marked NMT). This allows quantification of NMT mediated respiration for subtraction from all data. However in our experiments no oxygen consumption was recorded in this respiratory state, indicating that no non-mitochondrial oxygen consumption was detectable in our system using this strain background.

Typical respirometry profiles for wild type and $\Delta p d e 2$ cells cultured in the presence of $4 \mathrm{mM}$ cAMP are presented (Figure 2A). Significant differences were apparent between the respirometry profiles obtained for wild type and $\Delta p d e 2$ strains when grown in the presence of exogenous cAMP. While wild type cells typically demonstrated a routine respiration of around $6 \mathrm{pmols} / \mathrm{s} / 10^{6}$ cells under these conditions, cells lacking PDE2 showed a significant reduction, typically less than $2 \mathrm{pmols} / \mathrm{s} / 10^{6}$ cells (Figure 2A). This suggests that high levels of cAMP signaling suppress the respiratory activity of yeast mitochondria. To investigate this further we examined the respirometry profiles of wild type, $\Delta p d e 2$, $\Delta p d e 2 \Delta t p k 3$ and $\Delta p d e 2 \Delta t p k 3+T P K 3$ grown in the presence or absence of cAMP (Figure $2 \mathrm{~B}$ and summarised in Additional File 2). When cultured in the absence of cAMP both wild type and the double mutant ( $\Delta p d e 2 \Delta t p k 3)$ displayed similar routine respiration rates and maximal ETS levels upon FCCP addition. Interestingly the single mutant, $\Delta p d e 2$, showed a $20 \%$ increase in routine respiration and a maximal ETS that appeared higher than wild type when grown in the absence of additional cAMP (Figure 2B). Overexpression of TPK3 from a plasmid in $\Delta p d e 2 \Delta t p k 3$ resulted in a significant reduction in routine respiration, and notably cells failed to significantly increase respiration rate upon FCCP addition. In all strains the LEAK level of respiration appeared to be constant (Figure 2B).

The addition of $4 \mathrm{mM}$ cAMP did appear to have a small effect on respiratory activity in wild type cells using this highly sensitive assay. We observed a $17 \%$ reduction in routine respiration when compared to wild type cells cultured without cAMP. A similar reduction was observed in the LEAK level of respiration, but the maximal ETS level remained unchanged. When cells 
lacking $P D E 2$ were grown in the presence of exogenous cAMP we consistently observed a dramatic fall in routine respiration and maximal ETS rate (Figure 2C). Confirmation that Tpkp3 was responsible was obtained as respiratory activity was largely restored in $\Delta p d e 2 \Delta t p k 3$ cells grown under the same conditions (Figure 2C). Further evidence was obtained by the re-expression of $T P K 3$ in $\Delta p d e 2 \Delta t p k 3$ cells which resulted in the loss of respiration in the presence of exogenous cAMP. In addition, the addition of FCCP did not elevate respiration in either $\Delta p d e 2 \Delta t p k 3$ or $\Delta p d e 2 \Delta t p k 3+T P K 3$ cells, pointing to a loss of proton gradient across the inner mitochondrial membrane, further supporting the notion that elevated Tpkp3 activity leads to a loss of mitochondrial membrane potential. The data provided may also suggest that elevated levels of Tpk3p activity leads to reduction in the levels of complete and functional electron transport complexes.

\section{Effects of TPK3 on ROS production and mitochondrial morphology}

To establish whether elevated Tpk3p activity was sufficient to induce ROS production, wild type, $\Delta p d e 2$, $\Delta p d e 2 \Delta t p k 3$ and $\Delta p d e 2 \Delta t p k 3+T P K 3$ cultures were grown in the presence of the fluorescent indicator dye $\mathrm{H}_{2}$ DCF-DA. Samples were anlaysed by flow cytometry as well as visually under a fluorescent microscope (Figure $3 \mathrm{~A}$ ). In the presence of cAMP wild type cells were found to be generally unaffected, presumably as they were able to breakdown additional cAMP (data not shown). However the loss of Pde2p, and hence the ability to efficiently break down cAMP, resulted in a significant increase in ROS accumulating cells (Figure 3A). The additional loss of TPK3 in cells lacking PDE2 resulted in a significant reduction in the number of ROS positive population (Figure $3 \mathrm{~A}$ ). Importantly the reexpression of TPK3 on a plasmid in $\triangle p d e 2 \Delta t p k 3$ cells restored production of ROS (Figure $3 \mathrm{~A}$ ).

In order to investigate the effects of Tpk3p on mitochondrial morphology we made use of a plasmid that allows the expression of a mitochondrial targeted RFP protein (a kind gift from J. Shaw University of Utah) (Figure 3B). While a reticular network was seen in wildtype cells, mitochondria in $\Delta p d e 2$ mutant cells appeared reduced in number, were fragmented and visibly enlarged when grown in the presence of exogenous cAMP (Figure 3B). The abnormal mitochondria observed could be restored to a wild type appearance in cells lacking both PDE2 and TPK3 (Figure 3B). We also noted that in the $\Delta p d e 2$ mutant only a few enlarged structures of mtDNA could be seen when compared to the numerous structures visible in wild type (data not shown). In line with the improvement in mitochondrial morphology, the number of $\mathrm{mt}$ nucleoids was increased

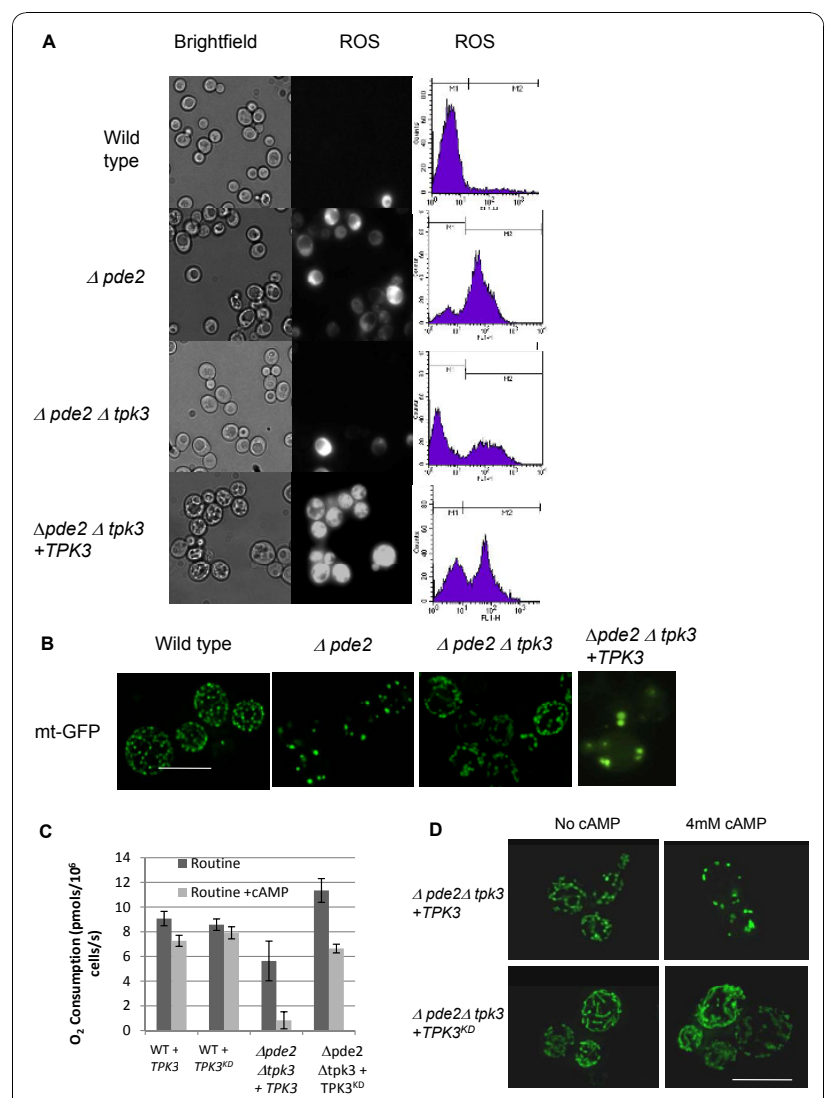

Figure 3 Tpk3p dependent ROS production and mitochondrial dysfunction. ROS production and aberrant mitochondrial morphology are a result of Tpk3p activity. We examined the effect on ROS production on diauxic shift cultures with and without high Tpk3p activity. Strains were grown in the presence of $4 \mathrm{mM}$ cAMP for 24 hours in MM supplemented with $\mathrm{H}_{2}$ DCF-DA. Cells were then either examined by fluorescence microscopy or flow cytometry for the presence of ROS, fluorescing cells that indicate the presence of ROS are found in the $M 2$ region of the histograms presented (A). Mitochondrial morphology was investigated by expression of a targeted GFP molecule (B). Routine respiration was assessed in wild type and $\triangle p d e 2 \triangle t p k 3$ cells overexpressing an active TPK3 or a kinase dead version (TPK3 ${ }^{K D}$ ) of the enzyme in the presence and absence of exogenous $4 \mathrm{mM}$ CAMP (C). Mitochondrial morphology was also assessed in $4 p d e 2 \Delta t p k 3$ cells overexpressing TPK3 or TPK $3^{K D}$ in the presence and absence of exogenous $4 \mathrm{mM}$ cAMP (D). Scale Bar = $10 \mu \mathrm{M}$.

in $\Delta p d e 2 \Delta t p k 3$ cells (data not shown). In addition fragmentation of genomic DNA (gDNA) was apparent in $\Delta p d e 2$ cells, a phenotype indicative of apoptosis. In contrast nuclear DNA fragmentation did not appear in $\Delta p d e 2 \Delta t p k 3$ cells (data not shown), consistent with our previous reports that Tpk3p induced mitochondrial dysfunction can trigger an apoptotic form of cell death $[27,30]$. In line with the ROS accumulation result (Figure $3 \mathrm{~A}$ ), the re-expression of TPK3 in $\triangle p d e 2 \Delta t p k 3$ was sufficient to restore the highly fragmented mitochondrial appearance observed in $\Delta p d e 2$ cells. 
To confirm that the kinase activity of Tpk3p is responsible for the respiratory and morphological defects observed in Dpde2 cells when cAMP levels are elevated we generated a kinase dead version of the enzyme. Deminoff et al (2006) demonstrated that the mutagenesis of residues $\mathrm{K} 336$ and $\mathrm{H} 338$ to alanine in the yeast PKA, Tpk1p produced a protein lacking kinase activity. As the catalytic domain of TPK1 and TPK3 are highly conserved, we mutagenised the equivalent K337 and $\mathrm{H} 339$ residues of Tpk3p to alanine. In line with these mutations rendering Tpk3p inactive, the expression of $T P K 3^{K D}$ in Dpde2Dtpk3 cells did not lead to the collapse of respiration (Figure $3 \mathrm{C}$ ) or to the manifestation of a highly fragmented mitochondrial network (Figure 3D). These data further suggest that elevated kinase activity of Tpk3p is sufficient to induce respiratory collapse and ROS production in yeast mitochondria.

\section{cAMP/Tpk3p Signalling regulates cell death in yeast colonies}

It has recently emerged that regulated cell death is employed within yeast colonies to facilitate multi-cellular communication and simple differentiation [41,42]. We therefore assessed the effects of Tpk3p activity on the patterning of cell death within colonies. We grew colonies for five days on YPD medium supplemented with $4 \mathrm{mM}$ cAMP and the cell death indicator Phloxine $\mathrm{B}$, which accumulates within dead or dying cells [27]. Wild type cells of this background display an accumulation of Phloxine B within the centre of the colony, which is most visible in the dissected cross section view presented in Figure 4. Colonies grown from cells lacking $P D E 2$ were smaller, disordered and accumulated phloxine $B$ throughout the colony, indicating widespread death. Small regions of phloxine B exclusion were

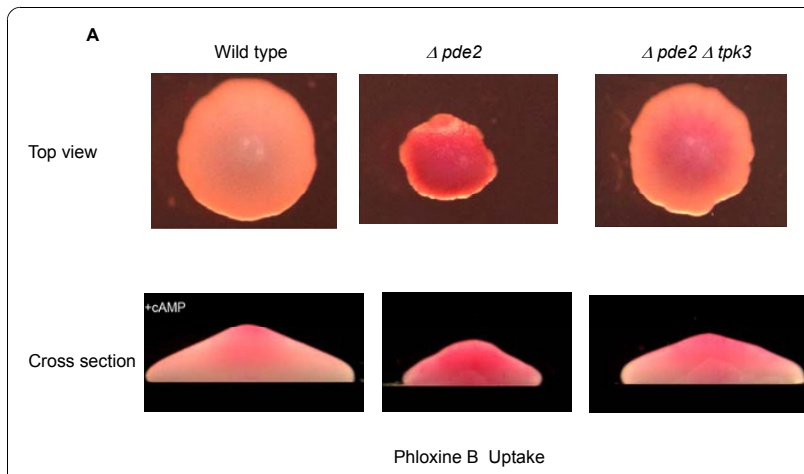

Figure 4 Elevated Tpk3p activity affects colony morphology. Cell death was visualized within growing yeast colonies grown for 5 days on rich media containing $10 \mu \mathrm{M}$ Phloxine B containing $4 \mathrm{mM}$ cAMP. Cells that are dead or dying accumulate the dye which stains them pink/red. Colonies were visualised from above before dissection and images captured incross section. apparent at the very growing edge of the colony. The deletion of TPK3 in $\triangle p d e 2$ cells resulted in a restoration of colony size and a central staining pattern that was slightly enlarged when compared to wild type but which represented a significant rescue of the cAMP induced colony death phenotype observed in single mutant $\Delta p d e 2$ colonies. These data suggest that cAMP/PKA signaling regulation of cell death plays a significant role in yeast colony patterning.

\section{Microarray analysis of changes in gene expression mediated by Tpk3p activity}

Our data indicate that elevated levels of Tpk3p activity have a profound effect on the respiratory capacity and function of yeast mitochondria. In an attempt to identify the regulatory mechanisms involved we conducted a microarray experiment. Single mutant $\Delta p d e 2$ and double mutant $\Delta p d e 2 \Delta t p k 3$ strains were grown to diauxic shift in the presence of $4 \mathrm{mM}$ cAMP, and mRNA levels analysed as described in materials and methods. Comparison of the data sets obtained allowed us to assess the impact of Tpk3p activity on transcriptional regulation. Two natural internal controls within the microarray data, namely the deletion of PDE2 and TPK3 in the single and double mutants compared to the wild type, allowed us to confirm the reliability of the data obtained. Expression of PDE2 in both the single and double mutant was $3.3 \%$ and $2.7 \%$ respectively compared to the wild type; TPK3 expression levels were $98 \%$ in the single $\Delta p d e 2$ mutant and $5.3 \%$ in the $\Delta p d e 2 \Delta t p k 3$ double mutant, confirming the genotype of the strains used in this study (Figure 5A). Interestingly expression levels of TPK2 were virtually unchanged in either mutant $(105 \%-\Delta p d e 2$ and $94 \%-\Delta p d e 2 \Delta t p k 3)$ whereas TPK1 levels were reduced to $60 \%$ of wild type levels in $\Delta p d e 2$ and $66 \%$ in the double mutant. In line with this Tpk1p protein levels were also markedly reduced in $\Delta p d e 2$ cells when grown in the presence of exogenous cAMP (data not shown). Of the 5800 transcripts screened, 118 were up-regulated by at least 2 fold and 124 down-regulated at least 2 fold when comparing $\Delta p d e 2$ and $\Delta p d e 2 \Delta t p k 3$ strains (listed in Additional files 3 and 4). Gene ontology anaylsis, using the GO-Slim Mapper software http://www.yeastgenome.org/cgi-bin/ $\mathrm{GO} /$ goSlimMapper.pl, allowed allocation of these genes to intracellular compartments (Figure 5B). We found that $41 \%$ of the down-regulated transcripts encoded mitochondrial genes. Closer inspection of the downregulated mitochondrial genes revealed a number of interesting targets that are involved directly in the mitochondrial ETS (Table 2). Both external mitochondrial NADH dehydrogenases, NDE1 and NDE2, which represent Complex I in yeast, were down regulated by 2 and 5 fold respectively. Similarly $S D H 2$, a component of 


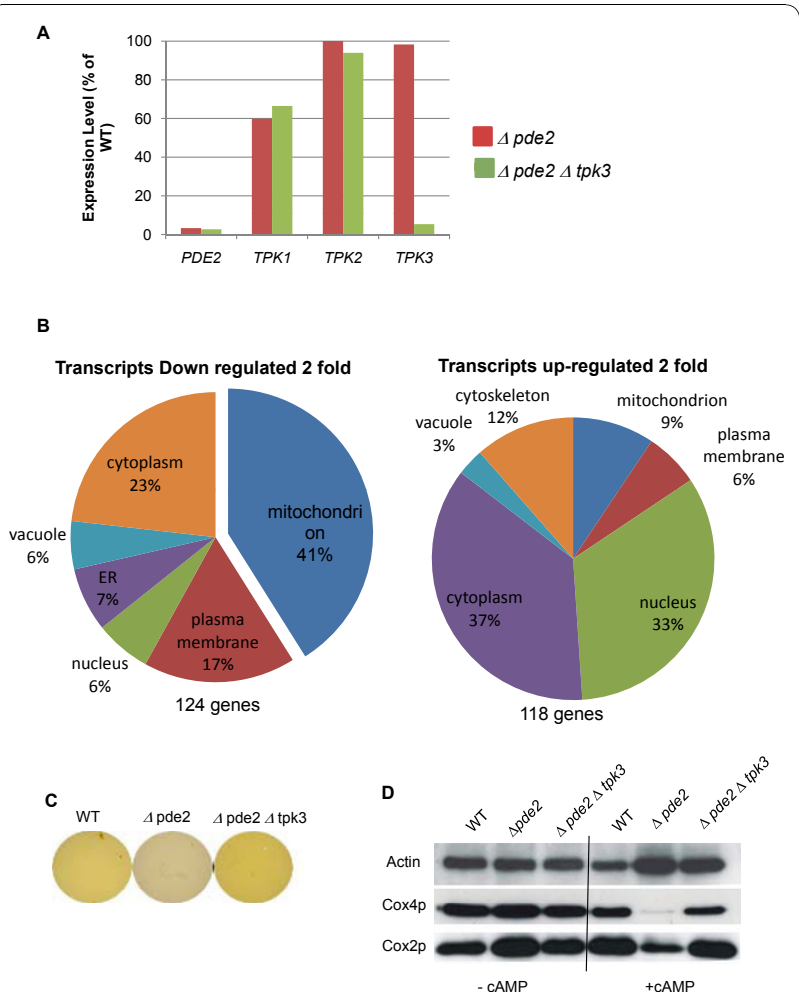

Figure 5 Microarray analysis of the effects of elevated Tpk3p activity. The effect of high Tpk3p activity on gene transcription was assessed. An affymatrix microarray was performed on RNA isolated from $4 p d e 2$ and $\Delta p d e 2 \triangle t p k 3$ strains grown to diaxuic shift in YPD media supplemented with $4 \mathrm{mM}$ CAMP. Confirmation of both the strains genotype and reliability of the micro array data can be seen by comparing expression levels of the effected genes PDE2 and TPK3: TPK1 and TPK2 expression levels are included for information (A). GOSlim Mapper software was used to identify likely intracellular compartments of genes either up or down regulated by at least 2 fold (B). Genes involved in glycogen biosynthesis were significantly down regulated in $\Delta p d e 2$ cells under these conditions but not in $\Delta p d e 2 \Delta t p k 3$. As further verification of the array data we assayed glycogen levels in wild type, $4 p d e 2$ and $\Delta p d e 2 \Delta t p k 3$ cells. In this assay a yellow colour is indicative of glycogen presence (C). Western blotting was used to assess the levels of the mitochondrial Cytochrome $c$ Oxidase protein Cox4p, Cox2p and actin (Act1p) in wild type, $4 p d e 2$ and $4 p d e 2 \Delta t p k 3$ cells grown in the presence or absence of $4 \mathrm{mM}$ CAMP. Protein equivalent to $2 \times 10^{6}$ cells was run on an SDS-PAGE gel and transferred to PVDF for immunodetection (D).

complex II - succinate dehydrogenase; $C Y T 1$ and RIP1 complex III subunits; CYC1-isoform-1 cytochrome c; COX4 a subunit of complex IV - Cytochrome c Oxidase and ATP19 a component of ATPsynthase were all down regulated. In addition, the expression of a number of regulatory components of the ETS was also reduced, including $C O Q 2$, which is required for ubiquinone biosynthesis; $C Y T 2$, an enzyme required for cytochrome c maturation; COX10 and SCO1, both required for Cytochrome c Oxidase formation and NCA3 which regulates expression of the mitochondrially encoded ATP synthase subunits 6 and 8 . In addition to the loss of crucial elements of the ETS the microarray revealed ROS detoxification enzymes (SOD1, SOD2 and CTT1) were not transcriptionally up-regulated despite the increase in ROS (Table 2).

Examination of up-regulated genes did not reveal any components of the ETS However genes involved in cellular respiration and mitochondrial regulation were identified (Table 3). These include PUF3, which links mitochondria to actin cables and is important for inheritance and morphological regulation [43]. Certain genes associated with cellular stress response were also upregulated in a Tpk3p dependent manner. For example an 8 fold increase in transcripts encoding the DNA damage response gene HUG1 was found. Interestingly, several transporters involved in glucose uptake, $H X T 1$, HXT2 and HXT11 were also upregulated (Table 3 and Additional File 3). These transporters are induced when the cell senses high glucose levels. Another gene that supported the idea that nutrient sensing in terms of glucose was compromised included MIG2 which has been shown to inhibit SUC2 expression in the presence of high glucose levels (Table 3).

A known feature of PKA activity is the ability to suppress the activation of biosynthesis of the storage carbohydrates glycogen and trehalose. This effect was noted in our array data. Several genes whose products are involved in glycogen and trehalose biosynthesis were downregulated in a Tpk3p dependent manner (Additional File 5). As a further control to verify the validity of our microarray data we investigated whether elevated Tpkp3 activity resulted in the suppression of glycogen accumulation as cells enter the stationary phase of growth. Wild type, $\Delta p d e 2$ and $\Delta p d e 2 \Delta t p k 3$ cells were grown for $48 \mathrm{~h}$ and stained for glycogen accumulation as described in materials and methods. Wild type cells start to accumulate glycogen at this point of growth (Figure 5C), however we found that cells lacking PDE2 fail to accumulate glycogen. This failure to accumulate glycogen could be reversed by the additional deletion of TPK3 (Figure 5C).

The microarray data also suggested that the elevation of cAMP levels leds to a Tpk3p dependent reduction in COX4 transcript levels, a core component of Cytochrome c Oxidase. We tested whether this observation could be re-capitulated at the protein level by conducting a western blot using wild type, $\Delta p d e 2$ and $\Delta p d e 2 \Delta t p k 3$ strains were grown to diauxic shift in the absence or presence of $4 \mathrm{mM}$ cAMP. In all strains grown without the addition of exogenous cAMP there appeared to be similar levels of Cox4p (Figure 5D). However upon addition of cAMP Cox4p was barely detectable in $\Delta p d e 2$ cells and a partial restoration was 
Table 2 Selected genes downregulated by elevated CAMP/TPK3 signalling

\begin{tabular}{llll}
\hline Function & Gene & Fold Change & Gene Function \\
\hline PKA & TPK1 & -1.1 & PKA catalytic subunit \\
& TPK2 & 1.1 & PKA catalytic subunit \\
\hline ETS & NDE1 & -2.0 & Mitochondrial external NADH dehydrogenase, catalyzes the oxidation of cytosolic NADH; \\
& NDE2 & -5.5 & Mitochondrial external NADH dehydrogenase, catalyzes the oxidation of cytosolic NADH; \\
& SDH2 & -2.3 & Iron-sulfur protein subunit of succinate dehydrogenase Complex II \\
& COQ2 & -2.0 & Catalyzes the second step in ubiquinone (coenzyme Q) biosynthesis \\
& RIP1 & -1.7 & Rieske Iron Sulphur Protein of Complex III \\
& CYT1 & -2.0 & Cytochrome c1, catalytic subunit of complex III \\
& CYT2 & -2.3 & Cytochrome c1 heme lyase, involved in maturation of CYT1 \\
& CYC1 & -1.9 & Cytochrome c, isoform 1; transfers electrons from Complex III to Complex IV \\
& COX4 & -2.2 & Subunit IV of cytochrome c oxidase (Complex IV) \\
& COX10 & -2.3 & Heme A:farnesyltransferase, required for cytochrome c oxidase activity; \\
& SCO1 & -2.0 & Copper-binding protein of the mitochondrial inner membrane, required for cytochrome c oxidase \\
& NCA3 & -6.2 & Regulates mitochondrial expression of subunits 6 (Atp6p) and 8 (Atp8p) of Complex V \\
& ATP19 & -2.2 & Subunit $k$ of the mitochondrial F1F0 ATP synthase \\
\hline Detox & SOD1 & -1.4 & Cytosolic superoxide dismutase \\
SOD2 & -1.1 & Mitochondrial superoxide dismutase; protects cells against oxygen toxicity \\
CT11 & -1.6 & Cytosolic catalase T, has a role in protection from oxidative damage by hydrogen peroxide
\end{tabular}

Selected genes involved in ETS and ROS detoxification that were downregulated in $\Delta p d e 2$ cells but not in $\Delta p d e 2 \Delta t p k 3$ when grown in the presence of exogenous $4 \mathrm{mM}$ cAMP. Gene lists were obtained as a ratio of statistically significant expression levels in $\Delta p d e 2 / \Delta p d e 2 \Delta t p k 3$ cells when grown in the presence of 4 mM cAMP.

observed in $\Delta p d e 2 \Delta t p k 3$ cells. As Cox4p is essential for complex IV stability, these data suggested that high levels of cAMP and Tpk3p activity may lead to a loss or respiration as a result of the loss of Cytochrome c oxidase from the mitochondria. In line with this the level of Cox $2 p$, which is encoded by the mitochondrial genome, was also greatly reduced upon cAMP elevation in $\Delta p d e 2$ cells (Figure 5D).

CAMP/PKA signaling regulates mitochondrial function and cell death via transcription factor activity

Our microarray data suggest that elevated TPK3 activity leads to considerable remodeling at the level of transcription, with particular emphasis on genes involved in mitochondrial function. In an attempt to identify transcription factors involved we utilised the bioinformatics analysis package Yeastract http://www.yeastract. $\mathrm{com} /$ to identify potential transcription factor involvement. Using this analysis software, which utilizes 48,333 characterised associations between known transcription factors and target genes, several candidate transcription factors were selected for further analysis, these were SOK2, SKO1 and HAP4 (Figure 6). The collapse of respiration observed in cells lacking $P D E 2$, when grown in the presence of $4 \mathrm{mM}$ cAMP, was partially rescued by the deletion of either SKO1 or SOK2 (Figure 6A). However in both cases a significant reduction in routine respiration rate was observed in response to cAMP addition. In contrast, although exhibiting a low starting routine rate of respiration, $\Delta p d e 2 \Delta$ hap 4 cells did not

Table 3 Selected genes uppregulated by elevated CAMP/TPK3 signalling

\begin{tabular}{llll}
\hline Function & Gene & $\begin{array}{l}\text { Fold } \\
\text { Change }\end{array}$ & Gene Function \\
\hline DNA damage & HUG1 & 8.0 & Responds to DNA damage or replication arrest, transcription is induced by DNA damage \\
& THI4 & 3.4 & Thiazole synthase, required for thiamine biosynthesis and for mitochondrial genome stability \\
\hline Nutrient sensing & HXT1 & 8.0 & $\begin{array}{l}\text { Glucose transporter, expressed in the presence of glucose, repressed when glucose is limited } \\
\text { Supressor of SUC2 expressed under high glucose levels }\end{array}$ \\
\hline Mitochondrial & PUF3 & 5.0 & OMM protein, linksArp2/3 complex with the mitochore promotes degradation of mRNAs for select nuclear- \\
Function & encoded mitochondrial proteins & \\
& IMC3 & 3.4 & maintenance of viability and in respiration
\end{tabular}

Genes involved in mitochondrial function, DNA damage response and nutrient sensing that were upregulated in $\Delta p d e 2$ cells but not in $\Delta p d e 2 \Delta t p k 3$ when grown in the presence of exogenous cAMP. Gene lists were obtained as a ratio of statistically significant expression levels in $\Delta p d e 2 / \Delta p d e 2 \Delta t p k 3$ cells when grown in the presence of $4 \mathrm{mM}$ cAMP. 


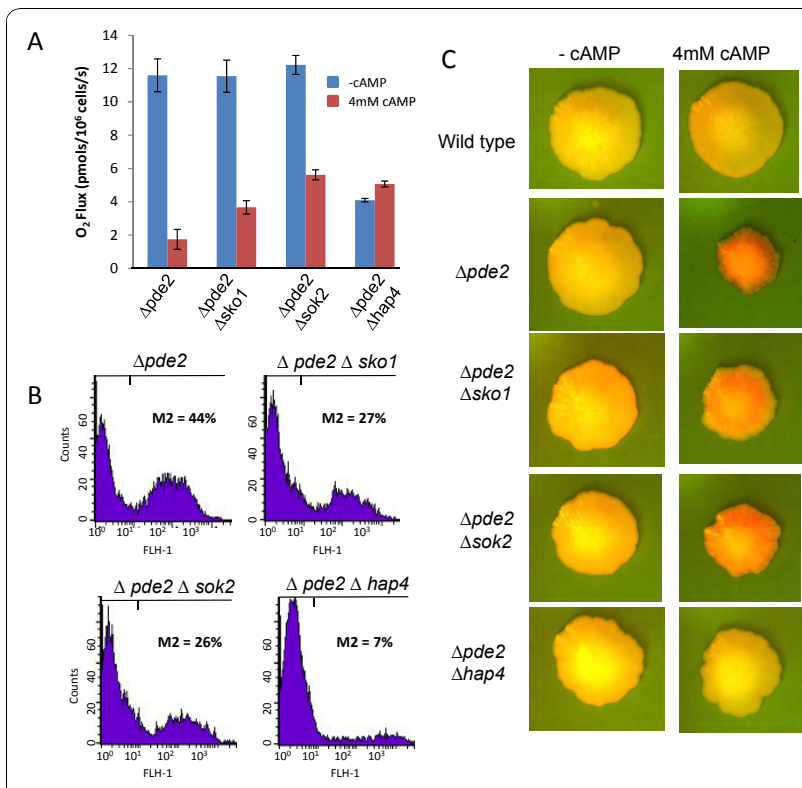

Figure 6 Influence of transcription factors predicted to play a role in TPK 3 based transcriptional remodeling under conditions of elevated CAMP. Cells lacking PDE2 and either of the transcription factors SKO1, SOK2 or HAP4 were examined for their capacity to regulate routine respiration, ROS production and cell death in response to CAMP addition. Routine respiration was measured in $\Delta p d e 2, \Delta p d e 2 \Delta s k o 1, \Delta p d e 2 \Delta s o k 2, \Delta p d e 2 \Delta h a p 4$ cells using a high resolution respirometer after growth for $24 \mathrm{~h}$ in YPD $+4 \mathrm{mM}$ CAMP (A). The same strains and growth conditions were used to assess the production of ROS using $\mathrm{H}_{2}$ DCF-DA (B) and the extent of cell death within colonies using phloxine $B$ uptake as described in materials and methods (C).

respond to the addition of cAMP (Figure 6A). In line with a partial restoration of mitochondrial function, both $\Delta p d e 2 \Delta s k o 1$ and $\Delta p d e 2 \Delta s o k 2$ showed a significant reduction in the number of cells accumulating high levels of ROS when compared to $\Delta p d e 2$ single mutant cells (Figure 6B). Strikingly the addition of cAMP to $\Delta p d e 2 \Delta$ hap4 did not induce significant ROS production (Figure 6B). We also examined the effects of cAMP elevation on cell death in colonies in these strains (Figure $6 \mathrm{C})$. We found that the loss of SKO1 or SOK2 in a $\triangle p d e 2$ background renders cells partially unresponsive to cAMP addition, resulting in increased colony size and reduced cell death as assessed by Phloxine $B$ uptake (Figure 6C). Cells lacking both PDE2 and HAP4 appeared to be resistant to cAMP exposure and did not exhibit signs of widespread cell death (Figure 6C).

\section{HAP4 activity leads to the CAMP/PKA dependent biogenesis of dysfunctional mitochondria prone to ROS production}

Our data points to a mechanism whereby cAMP induced ROS production and cell death occurs as a result of the modulation of multiple transcription factor activity. However the activity of HAP4 would appear to be of particular importance. It has been demonstrated that the HAP4 gene product is involved in stimulating mitochondrial biogenesis and elevating respiration during glucose de-repression, by enhancing the transcriptional activity of the HAP2/3/4/5 complex [44]. We therefore investigated the effects of HAP4 overexpression on mitochondrial function in wild type and $\Delta p d e 2$ cells grown in the presence of cAMP. To do this we overexpressed HAP4 from plasmids that utilized either an endogenous promoter or repressible Tetracycline, Tet $\mathrm{O}$, promoter. Both of these plasmids were able to fully restore the inability of a HAP4 deletion strain to grow on the non-fermentable carbon source glycerol, indicating that both could restore mitochondrial function (data not shown). Initially we examined the ability of HAP4 overexpression to suppress the mitochondrial morphology defects associated with high PKA activity. As both plasmids yielded exactly the same results we
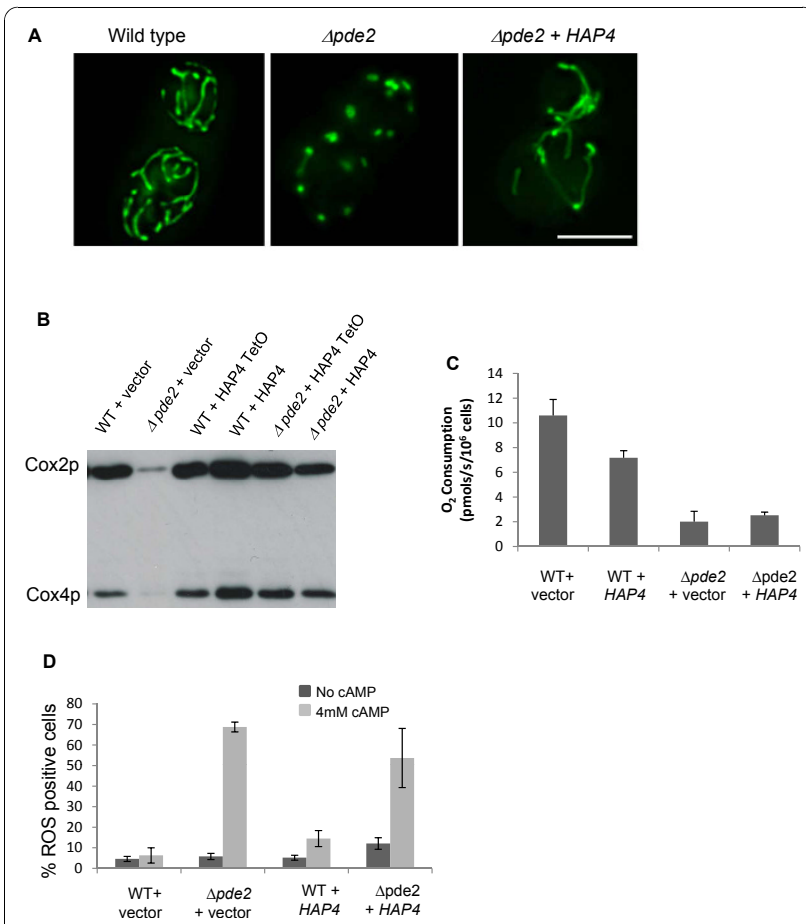

Figure 7 HAP4 overexpression leads to the production of dysfunctional mitochondria prone to ROS production

Mitochondrial morphology was examined in wild type + empty plasmid, $\triangle p d e 2+$ empty plasmid and $\triangle p d e 2+$ HAP4 cells grown to diauxic shift in minimal media supplemented with 4 mM cAMP using a mitochondria targeted GFP probe (A). The expression of nuclear and mitochondria encoded components of the Cytochrome c Oxidase complex (complex IV) was examined in wild type and $\triangle p d e 2$ cells grown in the presence of $4 \mathrm{mM}$ CAMP (B). The influence of HAP4 overexpression on the ability of wild type and 4 pde2 cells to respire (C) and to produce ROS (D) was examined under the same growth conditions. Scale Bar $=10 \mu \mathrm{M}$. 
present data for a single HAP4 expressing plasmid, that utilizing the Tet $\mathrm{O}$ promoter (Figure 7A). Mitochondria in cells lacking Pde2p develop fragmented and swollen mitochondria that fail to respire when grown in the presence of cAMP (Figure 7A and 3B). The overexpression of HAP4 was found to partially restore mitochondrial morphology to $\Delta p d e 2$ cells, in that a tubular array could be observed. However this network was never observed to be as elaborate as that seen in wild type cells (Figure 7A). The overexpression of HAP4 in $\triangle p d e 2$ cells was also able to increase the expression of nuclear (Cox $4 p$ ) and mitochondrial encoded (Cox2p) components of the Cytochrome c Oxidase complex (complex IV) of the electron transport chain (Figure 7B). This indicates that expression of $H A P 4$ is able to promote mitochondrial biogenesis in $\triangle p d e 2$ cells grown in the presence of cAMP. However the overexpression of HAP4 was insufficient to improve the poor oxygen consumption level observed in $\Delta p d e 2$ cells when treated with cAMP (Figure $7 \mathrm{~B})$. In addition, only a small reduction in the number of cells accumulating ROS could be observed when comparing $\triangle p d e 2$ cell grown in the presence of cAMP in the presence or absence of the HAP4 overexpression construct (Figure $7 \mathrm{C}$ ). It should be noted that the overexpression of HAP4 in wild type cells also resulted in a significant reduction in oxygen consumption (Figure 7B) and an increase in the number or ROS positive cells (Figure 7C). These results suggest that although increased expression of HAP4 can lead to an increase in mitochondrial biogenesis, it does not by itself lead to the production of healthy and fully functional organelles.

\section{Discussion}

The activity of cAMP/PKA signalling pathways appears to play an important role in the regulation of cell death in eukaryotes. For example it has been shown in lymphoid cells that elevated cAMP/PKA levels cause transcriptional changes that promote mitochondrial dependent apoptosis [45] andcan induce apoptosis via the up-regulation of Smac/DIABLO in cultured HeLa cells [46]. Our previous studies suggest that in yeast excessive PKA activity can also trigger, albeit via distinct signalling mechanisms to those observed to date in mammalian systems, mitochondria dependent apoptosis $[27,28,30]$. In yeast cAMP/PKA stimulated apoptosis involves the production of ROS from mitochondria. The mitochondrial state that leads to ROS production in actin aggregating cells requires PKA activity, and in particular the function of one of the three PKA subunits found in S. cerevisiae, Tpk3p [27,30]. Our data presented here supports a role for PKA signalling in regulating the activity of mitochondria via transcriptional control. In support of this our microarray analysis of the effects of cAMP elevation revealed a Tpk3p dependent suppression of a significant number of genes involved in mitochondrial function. Of particular note were genes whose products contribute to the electron transport system. The Tpk3p dependent downregulation of genes required for respiration also resulted in a significant reduction in respiratory capacity. Both the regulation of transcription and respiration could be restored by deletion of TPK3, implicating this single enzyme as an important regulator of mitochondrial biogenesis. Another possibility that must be considered is that Tpk3p localises to the mitochondria and regulates function directly via phosphorylation of protein targets. PKA activity within the mitochondria has been widely reported in mammalian systems and is known to modulate the activity of the respiratory chain [47]. Interestingly a fully functional carbon dioxide sensing cAMP/ PKA signalling system has recently been identified within human HeLa and COS-1 cell lines [48]. In yeast, the mitochondrial histone-like protein Abf2 has been shown to be phosphorylated by PKA, suggesting that activity of cAMP dependent phosphorylation also occurs within mitochondria of lower eukaryotes [49]. Phosphorylation of Abf2p by PKA was shown to inhibit its ability to interact with DNA, and the loss of $A B F 2$ resulted in mtDNA instability [49]. However fluorescence microscopy studies suggest that Tpk1p, Tpk2p and Tpk3p do not co-localise with the mitochondria (our unpublished results). In addition to this, the published mitochondrial proteome from S. cerevisae, despite identifying a number of protein kinases within the organelle, did not reveal the presence of PKA enzymes [50]. Further studies are required to fully address whether, as is the case in mammalian cells, cAMP/PKA signalling occurs within yeast mitochondria.

Further evidence that cAMP/Tpk3 signalling regulates mitochondrial biogenesis at the level of transcription comes from our finding that respiratory function, ROS production and cell death can be manipulated by rescued to varying degrees by the transcription factors SOK2, SKO1 and HAP4, which were predicted to play a role via bioinformatic analysis of our microarray data. Both SOK2 and SKO1 are transcription factors that have been previously implicated in cAMP signalling systems, and act as suppressor elements within stress signalling pathways. SOK2 is known to be regulated by cAMP/ PKA activity [51] and to play an important role in the regulation of cell death in colonies in response to ammonia signalling [42]. SKO1 is a repressor that mediates HOG pathway-dependent regulation by binding to cAMP response elements (CRE) sites in target promoters [52]. It is also known to play a regulatory role within cAMP/PKA stress signalling and can be phosphorylated by both HOG1 and PKA at independent sites [53]. It is likely that some of the toxicity associated with 
elevated cAMP/PKA activity occurs as a result of increased stress response suppression via the activity of SOK2 and SKO1. In line with this, the deletion of either $S O K 2$ or $S K O 1$ led to a partial restoration of respiratory activity and reduction in ROS production in $\Delta p d e 2$ cells grown in the presence of cAMP.

More significantly, the deletion of HAP4, a known mitochondrial biogenesis factor [54], prevented ROS production and cell death in $\Delta p d e 2$ cells grown in the presence of cAMP. This suggests that the activity of HAP4 present within cells experiencing high cAMP/ PKA signalling is responsible for the production of ROS. The overexpression of HAP4 led to the partial restoration of mitochondrial biogenesis in $\Delta p d e 2$ cells grown under conditions of cAMP elevation. However the additional mitochondria produced were not competent to respire and continued to produce high levels of ROS. We found that the overexpression of HAP4 in wild type cells also gave rise to mitochondria with reduced respiratory capacity, which were prone to ROS production. These data suggest that as yet unidentified factors aside from HAP4 are required to ensure the biogenesis of fully functional mitochondria. As the loss of TPK3 led to the restoration of fully functional mitochondria in $\Delta p d e 2$ cells grown under conditions of cAMP elevation it is likely that PKA function regulates mitochondrial activity via more than one effector to co-ordinate biogenesis in response to environmental change and metabolic demand. The search for further downstream targets of Tpk3p that are involved in mitochondrial regulation and biogenesis is currently underway. Elevation of Tpk3p activity also led to striking morphological defects, with mitochondria appearing fewer in number and as larger swollen structures. These mitochondria also exhibited reduced membrane potential and a reduction in respiratory chain components. Very similar features were reported to occur in mutants lacking Mdm38p and were attributed to the role that this protein plays in $\mathrm{K}+/ \mathrm{H}+$ exchange [55] raising the possibility that elevated Tpk3p induces a loss of homeostasis within the organelle.

Our observations suggest that elevated Tpkp3 activity induces a novel mitochondrial state that results in nonrespiring mitochondria prone to the production of ROS. A key question arising from this research is therefore, why do mitochondria damaged by elevated Tpk3p activity produce ROS? Interestingly a previous study has proposed that constitutive activation of the Ras pathway, via expression of the Ras ${ }^{\text {val19 }}$ allele, leads to a state 4 nonphosphorylating respiration that is prone to ROS production [16]. This study also suggested that the respiratory activity and mitochondrial membrane potential of Ras $^{\text {val19 }}$ cells becomes elevated, offering a plausible explanation as to the origin of ROS [16]. However in actin aggregating cells, which also exhibit constitutive Ras activation, and in $\Delta p d e 2$ cells grown in the presence of $4 \mathrm{mM}$ cAMP, there exists little or no mitochondrial membrane potential [27]. This was confirmed by treatment of cells with FCCP, a proton ionophore, that allows protons to flow back through the inner mitochondrial membrane into the matrix. This releases the inhibition caused by the build up of a proton gradient and allows the electron transport system to operate at its maximal rate (ETS). In cells with functional mitochondria, FCCP treatment normally results in an increase in oxygen consumption of around $30-40 \%$ (Figure $3 \mathrm{~A}$ ). However cells lacking PDE2 failed to respond to FCCP when grown in the presence of exogenous cAMP. This supports our suggestion that elevated Tpk3p activity results in a loss of mitochondrial membrane potential. We would argue that the constitutive activation of Ras/cAMP/PKA signalling can lead to altered mitochondrial function prone to ROS function that does not occur as a result of state 4 respiration.

\section{Conclusions}

In summary, our study has revealed that the loss of regulation of cAMP/PKA signalling leads to cell death that requires TPK3 dependent transcriptional remodelling that suppresses stress response signalling and promotes mitochondrial dysfunction. Tpk3p appears to facilitate cell death via action on several downstream targets, notably HAP4, which induce the formation of dysfunctional mitochondria which fail to respire and produce high levels of ROS. The loss of respiratory capacity within mitochondria has been associated with a wide range of human disorders including stroke, cardioencephalomyopathy, hepatic failure and Leigh's syndrome $[56,57]$ and is also associated with aging and agingrelated degenerative diseases such as Alzheimer's [58]. We hypothesise that the increased production of respiratory incompetent mitochondria that are prone to ROS production may contribute to the pathology of a variety of age related disease states. Further research in this area utilising yeast as a model eukaryote may well provide insights of important medical relevance.

\section{Additional material}

Additional file 1: Primers used in this study. Primers used to delete and confirm deletion of the genes TPK3, PDE2 and TPK1.

Additional file 2: TPK3 activity is required to promote loss of respiration. Respiratory profiles were determined for wild type, $\Delta p d e 2$, $\triangle$ pde2 $\triangle \mathrm{tpk} 3$ and $\triangle \mathrm{pde} 2 \triangle \mathrm{tpk} 3+$ TPK3 cells grown in the absence and presence of $4 \mathrm{mM}$ CAMP. Values of routine respiration (Routine), respiration facilitated by leak of protons across the inner membrane (LEAK), maximal respiratory rate (ETS), and respiratory control ratio (RCR) are the mean of 3 independent experiments, error bars are S.E. of those means. Values are for oxygen flux (pmols $/ \mathrm{s} / 10^{6}$ cells). 
Additional file 3: Genes down regulated by elevated CAMP/PKA activity. Complete list of genes down-regulated by 2 fold or more in $\Delta p d e 2$ cells but not in $4 p d e 2 \Delta t p k 3$ when grown in the presence of exogenous $4 \mathrm{mM}$ cAMP for $24 \mathrm{~h}$ to diauxic shift. Genes were grouped by GO assignment to a cellular process using the Slim Mapper tool as described in materials and methods.

Additional file 4: Genes up regulated by elevated cAMP/PKA activity. Complete list of genes up-regulated by 2 fold or more in $\Delta p d e 2$ cells but not in $\Delta p d e 2 \Delta t p k 3$ when grown in the presence of exogenous $4 \mathrm{mM}$ CAMP for $24 \mathrm{~h}$ to diauxic shift. Genes were grouped by GO assignment to a cellular process using the Slim Mapper tool as described in materials and methods.

Additional file 5: Effects of elevated CAMP/PKa activity on Carbohydrate storage genes. Genes involved in storage carbohydrate synthesis downregulated in $\Delta p d e 2$ cells but not in $\Delta p d e 2 \Delta t p k 3$ when grown in the presence of exogenous CAMP.

\section{Abbreviations}

(ETS): electron transport system; (TET): Triethyltin bromide; (FCCP): Carbonylcyanide-4-(trifluoromethoxy)-phenylhydrazone; (PKA): Protein Kinase A; (ROS): Reactive Oxygen Species

\section{Acknowledgements}

Thanks to Dr. Tobias von der Harr and Dr. G.U.Innes for critical reading of this manuscript and helpful comments. This work was sponsored by a Medical Research Council (MRC) career development fellowship to CWG (ref No. 78573$)$.

\section{Authors' contributions}

JEL carried out all of the high resolution respiromtery and ROS measurements included, and in collaboration with CWG made major contributions to every aspect of the paper including microscopy and microarray experiments. Both authors contributed to the conception of the study and to the writing and editing of the manuscript text.

Received: 1 September 2010 Accepted: 25 November 2010 Published: 25 November 2010

\section{References}

1. Butow RA, Avadhani NG: Mitochondrial Signaling: The Retrograde Response. Molecular Cell 2004, 14(1):1-15.

2. McBride HM, Neuspiel M, Wasiak S: Mitochondria: More Than Just a Powerhouse. Current Biology 2006, 16(14):R551-R560.

3. Wang C, Youle RJ: The Role of Mitochondria in Apoptosis. Annual review of genetics 2009.

4. Passos JF, Simillion C, Hallinan J, Wipat A, von Zglinicki T: Cellular senescence: unravelling complexity. Age Dordrecht, Netherlands 2009

5. Chan SL, Wei Z, Chigurupathi S, Tu W: Compromised respiratory adaptation and thermoregulation in aging and age-related diseases. Ageing research reviews 2009.

6. Turner N, Heilbronn LK: Is mitochondrial dysfunction a cause of insulin resistance? Trends in endocrinology and metabolism: TEM 2008, 19(9):324-330.

7. Gogvadze V, Orrenius S, Zhivotovsky B: Mitochondria as targets for chemotherapy. Apoptosis 2009, 14(4):624-640.

8. Mahad D, Lassmann H, Turnbull D: Review: Mitochondria and disease progression in multiple sclerosis. Neuropathology and applied neurobiology 2008, 34(6):577-589.

9. Gibson GE, Karuppagounder SS, Shi Q: Oxidant-induced changes in mitochondria and calcium dynamics in the pathophysiology of Alzheimer's disease. Annals of the New York Academy of Sciences 2008, 1147:221-232

10. Henchcliffe C, Beal MF: Mitochondrial biology and oxidative stress in Parkinson disease pathogenesis. Nature clinical practice 2008, 4(11):600-609.

11. Boveris A, Navarro A: Brain mitochondrial dysfunction in aging. IUBMB life 2008, 60(5):308-314.
12. Ishii N: Role of oxidative stress from mitochondria on aging and cancer. Cornea 2007, 26(9 Suppl 1):S3-9.

13. Orrenius S: Reactive oxygen species in mitochondria-mediated cell death. Drug metabolism reviews 2007, 39(2-3):443-455.

14. Hock MB, Kralli A: Transcriptional control of mitochondrial biogenesis and function. Annual review of physiology 2009, 71:177-203.

15. Ohlmeier S, Kastaniotis AJ, Hiltunen JK, Bergmann U: The Yeast Mitochondrial Proteome, a Study of Fermentative and Respiratory Growth. J Biol Chem 2004, 279(6):3956-3979.

16. Hlavata L, Aguilaniu H, Pichova A, Nystrom T: The oncogenic RAS2 $2^{\text {val19 }}$ mutation locks respiration, independently of PKA, in a mode prone to generate ROS. The EMBO journal 2003, 22(13):3337-3345.

17. Hlavata $L$, Nachin L, Jezek P, Nystrom T: Elevated Ras/protein kinase A activity in Saccharomyces cerevisiae reduces proliferation rate and lifespan by two different reactive oxygen species-dependent routes. Aging cell 2008, 7(2):148-157.

18. Reinders A, Burckert N, Boller T, Wiemken A, De Virgilio C: Saccharomyces cerevisiae cAMP-dependent protein kinase controls entry into stationary phase through the Rim15p protein kinase. Genes \& development 1998, 12(18):2943-2955.

19. Toda T, Cameron S, Sass P, Zoller M, Scott J, McCullen B, Hurwitz M, Krebs $E$, Wigler M: Cloning and characterisation of $B C Y 1$, a locus encoding a regulatory subunit of the cyclic AMP-dependent protein kinase in Saccharomyces cerevisiae. Molecular and cellular biology 1987, 7:1371-1377.

20. Toda T, Cameron S, Sass P, Zoller M, Wigler M: Three different genes in S. cerevisiae encode the catalytic subunits of the CAMP-dependent protein kinase. Cell 1987, 50:277-287.

21. Toda T, Uno I, Ishikawa T, Powers S, Kataoka T, Broek D, Cameron S, Broach J, Matsumoto K, Wigler M: In yeast, RAS proteins are controlling elements of adenylate cyclase. Cell 1985, 40(1):27-36.

22. Robertson LS, Causton HC, Young RA, Fink GR: The yeast A kinases differentially regulate iron uptake and respiratory function. Proceedings of the National Academy of Sciences of the United States of America 2000, 97(11):5984-5988.

23. Robertson LS, Fink GR: The three yeast $A$ kinases have specific signaling functions in pseudohyphal growth. Proceedings of the National Academy of Sciences of the United States of America 1998, 95(23):13783-13787.

24. Akada R, Yamamoto J, Yamashita I: Screening and identification of yeast sequences that cause growth inhibition when overexpressed. Mol Gen Genet 1997, 254:267-274.

25. Gerst JE, Ferguson $\mathrm{K}$, Vojtek A, Wigler M, Field J: CAP is a bifunctional component of the Saccharomyces cerevisiae adenylyl cyclase complex. Molecular and cellular biology 1991, 11(3):1248-1257.

26. Mintzer KA, Field J: Interactions between adenylyl cyclase, CAP and RAS from Saccharomyces cerevisiae. Cellular signalling 1994, 6(6):681-694.

27. Gourlay CW, Ayscough KR: Actin-Induced Hyperactivation of the Ras Signalling Pathway Leads to Apoptosis in Saccharomyces cerevisiae. Molecular and cellular biology 2006, 26(17):6487-6501.

28. Gourlay CW, Carpp LN, Timpson P, Winder SJ, Ayscough KR: A role for the actin cytoskeleton in cell death and aging in yeast. J Cell Biol 2004, 164(6):803-809.

29. Gourlay CW, Ayscough KR: Identification of an upstream regulatory pathway controlling actin-mediated apoptosis in yeast. J Cell Sci 2005, 118:2119-2132.

30. Leadsham JE, Miller K, Ayscough KR, Colombo S, Martegani E, Sudbery P, Gourlay CW: Whi2p links nutritional sensing to actin-dependent RasCAMP-PKA regulation and apoptosis in yeast. J Cell Sci 2009, 122:706-715.

31. Chevtzoff C, Vallortigara J, Averet N, Rigoulet M, Devin A: The yeast CAMP protein kinase Tpk3p is involved in the regulation of mitochondrial enzymatic content during growth. Biochem Biophys Acta 2005, 1706:117-125.

32. Gueldener U, Heinisch J, Koehler GJ, Voss D, Hegemann JH: A second set of loxP marker cassettes for Cre-mediated multiple gene knockouts in budding yeast. Nucleic acids research 2002, 30(6):e23.

33. Mazon MJ, Behrens MM, Morgado E, Portillo F: Low activity of the yeast CAMP-dependent protein kinase catalytic subunit Tpk3 is due to the poor expression of the TPK3 gene. European journal of biochemistry/FEBS 1993, 213(1):501-506.

34. Lascaris R, Bussemaker HJ, Boorsma A, Piper M, van der Spek H, Grivell L, Blom J: Hap4p overexpression in glucose-grown Saccharomyces 
cerevisiae induces cells to enter a novel metabolic state. Genome biology 2003, 4(1):R3.

35. von der Haar T: Optimized protein extraction for quantitative proteomics of yeasts. PloS one 2007, 2(10):e1078.

36. Gourlay CW, Carpp LN, Timpson P, Winder SJ, Ayscough KR: A role for the actin cytoskeleton in cell death and aging in yeast. J Cell Biol 2004, 164(6):803-809.

37. Westermann B, Neupert W: Mitochondria-targeted green fluorescent proteins: convenient tools for the study of organelle biogenesis in Saccharomyces cerevisiae. Yeast 2000, 16(15):1421-1427.

38. Wilson RB, Renault $G$, Jacquet $M$, Tatchell $K$ : The pde2 gene of Saccharomyces cerevisiae is allelic to rcal and encodes a phosphodiesterase which protects the cell from extracellullar CAMP. FEBS letters 1993, 325(3):191-195.

39. Nagley $P$, Hall RM, Ooi BG: Amino acid substitutions in mitochondrial ATPase subunit 9 of saccharomyces cerevisiae leading to oligomycin or venturicidin resistance. FEBS letters 1986, 195(1-2):159-163.

40. Cain K, Griffiths DE: Studies of energy-linked reactions. Biochem J 1977, 162:575-580

41. Vachova L, Devaux F, Kucerova H, Ricicova M, Jaca C, Palkova Z: Sok2p transcription factor is involved in adaptive program relevant for long term survival of Saccharomyces cerevisiae colonies. The Journal of biological chemistry 2004, 279(36):37973-37981.

42. Vachova L, Palkova Z: Physiological regulation of yeast cell death in multicellular colonies is triggered by ammonia. The Journal of cell biology 2005, 169(5):711-717.

43. Garcia-Rodriguez L, Gay AC, Pon LA: Puf3p, a Pumilio family RNA binding protein, localizes to mitochondria and regulates mitochondrial biogenesis and motility in budding yeast. The Journal of cell biology 2007, 176(2):197-207.

44. Forsburg SL, Guarente L: Identification and characterization of HAP4: a third component of the CCAAT-bound HAP2/HAP3 heteromer. Genes \& development 1989, 3(8):1166-1178.

45. Zhang L, Zambon AC, Vranizan K, Pothula K, Conklin BR, Insel PA: Gene expression signatures of CAMP/protein kinase A (PKA)-promoted, mitochondrial-dependent apoptosis. Comparative analysis of wild-type and cAMP-deathless $\mathbf{S} 49$ lymphoma cells. The Journal of biological chemistry 2008, 283(7):4304-4313.

46. Martinez-Velazquez M, Melendez-Zajgla J, Maldonado V: Apoptosis induced by cAMP requires Smac/DIABLO transcriptional upregulation. Cellular signalling 2007, 19(6):1212-1220.

47. Pagliarini DJ, Dixon JE: Mitochondrial modulation: reversible phosphorylation takes center stage? Trends in biochemical sciences 2006 31(1):26-34

48. Acin-Perez R, Salazar E, Kamenetsky M, Buck J, Levin LR, Manfredi G: Cyclic AMP produced inside mitochondria regulates oxidative phosphorylation. Cell metabolism 2009, 9(3):265-276.

49. Cho JH, Lee YK, Chae CB: The modulation of the biological activities of mitochondrial histone Abf2p by yeast PKA and its possible role in the regulation of mitochondrial DNA content during glucose repression. Biochimica et biophysica acta 2001, 1522(3):175-186.

50. Sickmann A, Reinders J, Wagner Y, Joppich C, Zahedi R, Meyer HE, Schonfisch B, Perschil I, Chacinska A, Guiard B, et al: The proteome of Saccharomyces cerevisiae mitochondria. Proceedings of the National Academy of Sciences of the United States of America 2003, 100(23):13207-13212

51. Ward MP, Gimeno CJ, Fink GR, Garrett S: SOK2 may regulate cyclic AMPdependent protein kinase-stimulated growth and pseudohyphal development by repressing transcription. Molecular and cellular biology 1995, 15(12):6854-6863.

52. Rep M, Proft M, Remize F, Tamas M, Serrano R, Thevelein JM, Hohmann S: The Saccharomyces cerevisiae Sko1p transcription factor mediates HOG pathway-dependent osmotic regulation of a set of genes encoding enzymes implicated in protection from oxidative damage. Molecular microbiology 2001, 40(5):1067-1083.

53. Pascual-Ahuir A, Posas F, Serrano R, Proft M: Multiple levels of control regulate the yeast CAMP-response element-binding protein repressor Sko1p in response to stress. The Journal of biological chemistry 2001, 276(40):37373-37378.

54. Lascaris R, Piwowarski J, van der Spek H, Teixeira de Mattos J, Grivell L, Blom J: Overexpression of HAP4 in glucose-derepressed yeast cells reveals respiratory control of glucose-regulated genes. Microbiology (Reading, England) 2004, 150(Pt 4):929-934.

55. Nowikovsky K, Reipert S, Devenish RJ, Schweyen RJ: Mdm38 protein depletion causes loss of mitochondrial $\mathrm{K}+/ \mathrm{H}+$ exchange activity, osmotic swelling and mitophagy. Cell death and differentiation 2007, 14(9):1647-1656

56. Barrientos A, Barros MH, Valnot I, Rotig A, Rustin P, Tzagoloff A. Cytochrome oxidase in health and disease. Gene 2002, 286(1):53-63.

57. Shoubridge EA: Cytochrome c oxidase deficiency. American journal of medical genetics 2001, 106(1):46-52.

58. Sullivan PG, Brown MR: Mitochondrial aging and dysfunction in Alzheimer's disease. Progress in neuro-psychopharmacology \& biological psychiatry 2005, 29(3):407-410.

doi:10.1186/1471-2121-11-92

Cite this article as: Leadsham and Gourlay: CAMP/PKA signaling balances respiratory activity with mitochondria dependent apoptosis via transcriptional regulation. BMC Cell Biology 2010 11:92.

\section{Submit your next manuscript to BioMed Central and take full advantage of:}

- Convenient online submission

- Thorough peer review

- No space constraints or color figure charges

- Immediate publication on acceptance

- Inclusion in PubMed, CAS, Scopus and Google Scholar

- Research which is freely available for redistribution 